\title{
Fast 4D Flow MRI Re-Emerges as a Potential Clinical Tool for Neuroradiology
}

S hortly after the introduction of clinical MR imaging, Moran ${ }^{1}$ demonstrated that velocity and flow could be measured noninvasively by using flow-encoding gradients integrated into conventional MR imaging techniques. This innovation was quickly implemented, resulting in $2 \mathrm{D}$ and $3 \mathrm{D}$ phase-contrast MRA. ${ }^{2}$ The value of $4 \mathrm{D}$ flow MR imaging for the evaluation of brain AVMs was recognized by early investigators. ${ }^{3}$ However, initial excitement in 4D flow MR imaging was dampened by low resolution, loss of signal due to complex flow, difficulty selecting the velocity encoding, and the long scanning times for $4 \mathrm{D}$ acquisition. Fortunately, many of these limitations have been overcome, and we are entering a new era of fast high-resolution 4D flow MR imaging. Acquisition times have been reduced by using strategies such as compressed sensing ${ }^{4}$ and radial $k$-space trajectories. ${ }^{5}$ Shorter TEs have reduced signal loss, and new encoding strategies improve the dynamic range of velocities that are detected. ${ }^{6}$ 3T scanners and 32channel coils provide substantial increases in signal and signal detection, enabling higher spatial resolution examinations. ${ }^{7}$ These improvements have already enabled 4D flow MR imaging to become a viable clinical tool in the assessment of a variety of non-neurologic conditions such as diseases of the aortic arch, ${ }^{8}$ congenital heart disease, ${ }^{9}$ portal venous hypertension, ${ }^{10}$ and renal arterial stenosis. ${ }^{11}$

In this issue of $A J N R$, Ansari et $\mathrm{al}^{12}$ have demonstrated that $4 \mathrm{D}$ flow MR imaging may play an important role in neurovascular diseases such as brain AVMs. The report demonstrated that cardiac-gated 4D flow MR imaging of the entire brain can be performed with excellent spatial and temporal resolution. The power of $4 \mathrm{D}$ flow MR imaging is that it allows simultaneous measurement of flow in the entire cerebrovascular system throughout the cardiac cycle, encompassing both arteries and veins. ${ }^{13}$ The $4 \mathrm{D}$ flow examination provides magnitude images that display the vascular anatomy and $3 \mathrm{D}$ velocity flow fields that can be used to derive flow-path ${ }^{14}$ and to estimate important parameters such as wall shear stress ${ }^{15}$ and pressure gradients. ${ }^{16}$ Ansari et al showed how $4 \mathrm{D}$ flow MR imaging can be used to improve the characterization of brain AVMs by incorporating physiologic information into the imaging assessment. It is now possible to extend the characterization to not only include important anatomic features such as size, location, and vascular components of arterial supply and drainage patterns, but also the flow conditions within each major arterial feeder, arteries near the AVM, and contralateral arteries permitting a global assessment of flow across the entire cerebrovascular network.

The flow network can be further defined by generating velocity-derived flow-path lines providing an overview of the dominant flow channels. Future applications will likely provide quantitative measurements of flow, estimates of wall shear stress, and pressure gradients. The vascular anatomy can be eloquently displayed by using the velocity data within each voxel to derive streamlines weighted by the distance traveled per second. Virtual MR cartography requires segmentation of vessel boundaries followed by manual positioning of the plane emitter by using vessel cross-sections and blood-flow tracking within these vessels by generating velocity-based selective streamlines. A selective cartography of the vascular malformation can be displayed by choosing the starting point of the flow-tracking. The virtual selective cartography has the potential to improve the anatomic characterization and aid in pretreatment planning. ${ }^{17}$

Ansari et al showed the ability of $4 \mathrm{D}$ flow MR imaging to assist in the selection of vessels to treat and to monitor flow conditions during staged embolization. The arterial components are differentiated by the size, flow volume, velocity profile, and flow features and can be compared to relatively normal contralateral vessels. The alterations in flow during staged embolization are easily appreciated and in some instances may improve targeting of vessels for treatment.

However, although the flow information is very relevant, it is yet to be determined whether it can significantly contribute to the selection of patients for open surgical resection, endovascular surgery, radiosurgery, and combined therapy or provide information regarding risk stratification. The challenges for future work are how to apply this technology to enhance treatment and improve outcomes. Targets for the technology might include better definitions of steal phenomenon, the influence of the AVM on global brain flow, and the impact of steal on autoregulation. A somewhat less explored component is venous drainage; $4 \mathrm{D}$ flow MR imaging can show high-velocity flow in arterialized veins, identify venous stenosis, measure pressure gradients, and quantitate arteriovenous shunts. Another application may be the detection of high- 
risk flow conditions that may promote aneurysm formation in arteries that supply the AVM. ${ }^{18}$

Currently, a significant limitation in the development of $4 \mathrm{D}$ flow MR imaging is the lack of easy-to-use postprocessing software tools that are readily available in a clinical environment. Most groups engaged in 4D flow MR imaging have elaborate inhouse software libraries that require substantial time and effort to create meaningful flow data. Although there are many avenues to explore based on derivatives of the velocity vector fields (eg, streamlines, path lines, particle path traces, relative wall shear stress, pressure gradients), without appropriate software tools, these will remain in the research domain.

Combining time-resolved contrast-enhanced MRA (4D tr CE MRA) and 4D flow MR imaging into 1 acquisition is also possible when highly undersampled radial acquisition is used to dramatically reduce the imaging time. ${ }^{19,20}$ This approach, called HYPRFlow, provides time-resolved contrast-enhanced whole-brain serial images that display the vascular anatomy with good temporal and spatial resolution and also generate cardiac-synchronized 3D velocity flow fields. The entire highly accelerated examination takes slightly $>5$ minutes. Recently, investigators have shown that $4 \mathrm{D}$ tr CE MRA can also be analyzed to generate color-coded time-of-arrival maps that improve delineation of the AVM arterial supply and reveal variable intranidal flow patterns. ${ }^{21}$

Finally, although highly controversial, 4D flow MR imaging can, in fact, assist in the characterization of intracranial aneurysms based on flow features such as a compact inflow jet, a small focal impingement zone, high vorticity, and wall shear stress, which have been identified as possibly contributing to aneurysm rupture. $^{22}$ There is still a great deal of debate on which flow features are important and how they contribute to aneurysm formation, growth, and rupture; however, it is likely that ultra-highresolution $4 \mathrm{D}$ flow MR imaging will provide information similar to computational fluid dynamics and will contribute to the hemodynamic assessment of intracranial aneurysms. ${ }^{23}$

$4 \mathrm{D}$ flow MR imaging will have a role to play in the evaluation of neurovascular diseases. The challenge will be how to apply the technology to provide clinically useful information with well-defined applications.

\section{REFERENCES}

1. Moran PR. A flow velocity zeugmatographic interlace for NMR imaging in humans. Magn Reson Imaging 1982;1:197-203

2. Pelc NJ, Bernstein MA, Shimakawa A, et al. Encoding strategies for three-direction phase-contrast MR imaging of flow. J Magn Reson Imaging 1991;1:405-13

3. Marks M, Pelc N, Ross M, et al. Determination of cerebral blood flow with a phase contrast cine MR imaging technique: evaluation of normal subjects and patients with arteriovenous malformations. Radiology 1992;182:467-76

4. Stadlbauer A, van der Riet W, Creleir G, et al. Accelerated time resolve three dimensional MR velocity mapping of blood flow patterns in the aorta using SENSE and k-t BLAST. Eur J Radiol 2010;75:e15-21

5. Gu T, Korosec F, Block W, et al. PC VIPR: a high speed 3D phase contrast method for flow quantification and high resolution angiography. AJNR Am J Neuroradiol 2005;26:743-49

6. Johnson K, Markl M. Improved SNR in phase contrast velocimetry with five point balanced flow encoding. Magn Reson Med 2010;63: 349-55
7. Wetzel S, Meckel S, Frydrychowicz A, et al. In vivo assessment and visualization of intracranial arterial hemodynamics with flow sensitized 4D MR imaging at 3T. AJNR Am J Neuroradiol 2007;28:433-38

8. Frydrychowicz A, François CJ, Turski PA. Four-dimensional phase contrast magnetic resonance angiography: potential clinical applications. Eur J Radiol 2011;80:24-35

9. Markl M, Geiger J, Kilner P, et al. Time resolved three-dimensional magnetic resonance velocity mapping of cardiovascular flow paths in volunteers and patients with Fontan circulation. Eur J Cardiothorac Surg 2011;39:206-12

10. Roldán-Alzate A, Frydrychowicz A, Niespodzany E, et al. In vivo validation of 4D flow MRI for assessing the hemodynamics of portal hypertension. J Magn Reson Imaging 2013;37:1100-08

11. François C, Lum D, Johnson K, et al. Renal arteries: isotropic, highspatial-resolution, unenhanced MR angiography with three-dimensional radial phase contrast. Radiology 2011;258:254-60

12. Ansari SA, Schnell S, Carroll T, et al. Intracranial 4D flow MRI: toward individualized assessment of arteriovenous malformation hemodynamics and treatment-induced changes. AJNR Am J Neuroradiol 2013 May 2 [Epub ahead of print]

13. Hope M, Percell D, Hope T, et al. Complete intracranial arterial and venous blood flow evaluation with $4 \mathrm{D}$ flow MR Imaging. AJNR Am J Neuroradiol 2009;30:362-66

14. Markl M, Kilner P, Ebbers T. Comprehensive 4D velocity mapping of the heart and great vessels by cardiovascular magnetic resonance. J Cardiovasc Magn Reson 2011;13:7-12

15. Boussel L, Rayz v, McCulloch C, et al. Aneurysm growth occurs at region of low wall shear stress: patient-specific correlation of hemodynamics and growth in a longitudinal study. Stroke 2008;39:2997-3002

16. Lum DP, Johnson KM, Paul R, et al. Transstenotic pressure gradients: measurements in swine: ECG gated 3D phase contrast MRA versus endovascular pressure sensing guidewires. Radiology 2007;245:751-60

17. Roca P, Edjlali M, Rabrait C, et al. Selective MR flow cartography of brain vascular malformations. In: Proceedings of the 21st Annual Meeting of the International Society for Magnetic Resonance in Medicine, Salt Lake City, Utah. April 20-26, 2013

18. Chang W, Loecher MW, Wu Y, et al. Hemodynamic changes in patients with arteriovenous malformations assessed using high resolution 3D radial phase contrast MR angiography. AJNR Am J Neuroradiol 2012;33:1565-72

19. Velikina J, Johnson K, Wu Y, et al. PC HYPRFlow: a technique for rapid imaging of contrast dynamics. J Magn Reson Imaging 2010;31:447-56

20. Grist T, Mistretta C, Strother C, et al. Time-resolved angiography: past, present and future. J Magn Reson Imaging 2012;36:1273-86

21. Illies T, Forkert ND, Ries T, et al. Classification of cerebral arteriovenous malformations and intranidal flow patterns by color-encoded 4D hybrid MRA. AJNR Am J Neuroradiol 2013;34:46-53

22. Boussel L, Rayz V, Martin A, et al. Phase-contrast magnetic resonance imaging measurements in intracranial aneurysms in vivo of flow patterns, velocity fields, and wall shear stress: comparison with computational fluid dynamics. Magn Reson Med 2009;61:409-17

23. Kecskemeti S, Johnson KJ, Wu Y, et al. High resolution three dimensional cine phase contrast MRI of small intracranial aneurysms using a stack of stars k-space trajectory. J Magn Reson Imaging 2012;35:518-27

P. Turski

University of Wisconsin School of Medicine Madison Wisconsin M. Edjlali C. Oppenheim Sainte-Anne Hospital Center Paris, France

http://dx.doi.org/10.3174/ajnr.A3664 